\title{
LA PALEONTOLOGÍA DE VERTEBRADOS EN CANARIAS
}

\author{
Carolina CASTILLO', Mercedes LÓPEZ1, Mercedes MARTÍN² y Juan \\ Carlos RANDO ${ }^{1}$
}

\author{
${ }^{1}$ Departamento de Biología Animal. Universidad de La Laguna, 38026 La Laguna, Tenerife. \\ 2 Instituto Canario de Paleopatología y Bioantropología. Aptdo. Correos 853, 38080 Santa Cruz de Tenerife.
}

Castillo, C., López, M., Martín, M. y Rando, J.C. 1996 La paleontología de vertebrados en Canarias. [The vertebrate paleontology in Canary Islands]. Revista Española de Paleontología, No Extraordinario, 237-247. ISSN 0213-6937.

\begin{abstract}
The Canary Islands are located at the NW of Africa. They are volcanic in origin. The geological characteristics of the archipelago are conditioning of the sites favourable for the preservation of fossilized remains which most importants are volcanic caves. Other sites are located in volcanic tuff, fossil dunes, debris avalanche deposits and reased beaches.

The fossil remains of vertebrate in the archipelago are fishes, reptils, birds and mammals. Amphibians were introduced by men.

There are several examples of gigantism among the fossil fauna of the Canary Islands (Gallotia and Canariomys) as well as a certain trend to apterism among birds.

Several items have been treated during the paleontological research: description, origin, phylogenetic relationships, morphological adaptation, biostratigraphic and biochronology approach. It has been described eigth endemic terrestrial reptiles species, four endemic terrestrial mammals species, two endemic terrestrial bird species and two species of shearwaters. The fossil record of vertebrates range from Miocene to Holocene.

In this paper we make a review of the present status and legislation on Paleontology in the archipelago and the scarce protection that the paleontological sites have in the islands.
\end{abstract}

Keywords: historic review, fossil vertebrates, present status, legislation, Canary Islands.

\section{RESUMEN}

Las Islas Canarias se localizan geográficamente al NO del continente africano. Su origen es volcánico. Las características geológicas del Archipiélago condicionan los tipos de yacimientos favorables a la preservación de los restos fósiles. Los más importantes son los tubos volcánicos. Otros yacimientos se encuentran en tobas volcánicas, dunas fósiles, derrubios de laderas y playas fósiles.

Los restos fósiles de vertebrados conocidos en el archipiélago pertenecen a peces, reptiles, aves y mamíferos, faltando los anfibios que fueron introducidos por el hombre.

En Canarias la fauna fósil de vertebrados presenta algunos ejemplos de gigantismo (Gallotia y Canariomys), así como cierta tendencia al apterismo en aves.

Los aspectos tratados en las investigaciones paleontológicas han sido, entre otros, la descripción, el origen, relaciones filogenéticas, las adaptaciones morfológicas de las distintas especies y la aproximación bioestratigráfica y biocronológica. Se han descrito dieciséis especies endémicas, de ellas ocho corresponden a reptiles terrestres, cuatro a mamíferos y cuatro a aves. El registro fósil de vertebrados se extiende desde el Mioceno al Holoceno.

En este trabajo se hace una revisión de las investigaciones realizadas y de la legislación vigente sobre Paleontología en el Archipiélago, y se trata la problemática de la falta de protección de los yacimientos.

Palabras clave: síntesis histórica, vértebrados fósiles, estado actual, legislación, Islas Canarias.

\section{INTRODUCCIÓN}

Las islas Canarias se localizan geográficamente al NO del continente africano, a $28^{\circ}$ de latitud $\mathrm{N}$ y de $13^{\circ}$ a $18^{\circ}$ de longitud $\mathrm{O}$, aproximadamente, e incluye siete islas principales y varios islotes. Lanzarote y Fuerteventura son las islas más antiguas y El Hierro la más moderna: 15,5 Ma y 20,4 Ma (Coello et al., 1992) y 0,8 Ma (Fúster et al., 1993) respectivamente, para los materiales subaéreos. Los primeros fósiles descritos procedentes de formaciones marinas sedimentarias, corresponden al Cretácico inferior (Puerto de la Peña, Fuerteventura; Renz et al., 1992), aunque en la base de estas formaciones se ha citado la presencia de impresiones de Posidonia de edad jurásica (Rothe, 1968). Los restos hallados en las formaciones continentales pertenecen al Mioceno (Orzola, Lanzarote; Rothe, 1964; GarcíaTalavera 1990).

En estas islas se conocen fósiles y subfósiles de todos los grupos de vertebrados excepto los anfibios que han sido introducidos después de la llegada de los europeos; los peces son el grupo menos estudiado a nivel paleontológico. En general, el estudio de los vertebrados constituye una importante fuente de información sobre la colonización, evolución y extinción en islas.

La investigación en Paleontología de vertebrados en el Archipiélago no cobró auge hasta la década de los ochenta, y 
esto posiblemente se debiera a la concepción de las dificultades que conlleva la conservación de los restos fósiles en el sustrato volcánico que constituye las islas.

El registro fósil de vertebrados en Canarias en el estado actual de nuestro conocimiento, se extiende desde el Mioceno al Holoceno, aunque de forma bastante discontinua. La mayor parte de los restos se incluyen en el Pleistoceno superior y en el Holoceno.

\section{SITUACIÓN GEOLÓGICA}

Existen varias teorías sobre el origen y génesis del Archipiélago que están actualmente en debate; unas hacen referencia al carácter oceánico o continental de la corteza subyacente y otras, a la génesis de la fracturación ligada al volcanismo, en relación con la apertura del Atlántico o con la Tectónica del Norte de Africa. De forma general se acepta la existencia de corteza oceánica bajo las islas, aunque con un carácter transicional en las islas más orientales (Hernán Reguera, 1993-95)

\section{Fuenteventura y Lanzarote}

Fuerteventura y Lanzarote son parte de un sistema montañoso paralelo a la costa africana. Desde el punto de vista volcanoestratigráfico se distinguen dos formaciones principales: el Complejo Basal y las Series volcánicas subaéreas (Coello et al., 1992).

Las series volcánicas subaéreas están comprendidas en dos grandes conjuntos: Serie I, discordante con el Complejo Basal y cuya base es Miocena; Series II, III y IV desde el Plioceno hasta la época reciente. La datación más antigua de los materiales subaéreos de Lanzarote es de 15,5 \pm 0,3 Ma. En Fuerteventura, los materiales subaéreos más antiguos tienen una edad de 20,4 \pm 0,4 Ma (Coello et al., op. cit.).

\section{Gran Canaria}

La historia geológica de Gran Canaria se remonta al Mioceno medio. El crecimiento de la isla se hace en una sola fase rápida con episodios destructivos significativos. La parte subaérea de la isla fue construida por tres ciclos magmáticos principales, que según Pérez Torrado (1992) ocurrieron del modo siguiente: el Ciclo I o antiguo tuvo lugar entre los 14 Ma y 8,5 Ma, al que siguió un período de inactividad que afectó a toda la isla; el Ciclo II corresponde a la formación Roque Nublo, desarrollada entre 5,3 y 2,9 Ma, y la tercera reactivación volcánica (Ciclo III) ocurre desde los 2,9 Ma hasta tiempos subrecientes $(3.075 \pm 50$ a BP); entre la segunda y tercera fases magmáticas principales tuvo lugar un período de inactividad que sólo afectó a las zonas costeras y de medianías, mientras que la actividad volcánica continuaba en el resto de la isla.

\section{Tenerife}

La serie volcánica más antigua corresponde al Mioceno tardío y aparece en Anaga (NE), Teno (NO) y Roque del Conde (S). Ancochea et al. (1990) distinguen: en Anaga, series basálticas antiguas, con tres ciclos volcánicos datados en 6,5 Ma, 6,5-4,5 Ma y 3,6 Ma; en Teno, con dos ciclos de actividad entre 6,7 y 4,5 Ma, y dos series principales separadas por una posible pausa entre 6,2 y 5,6 Ma; y en Roque del Conde, con dos ciclos entre 11,6 Ma y 3,5 Ma. Entre los 3,3 y 1,9 Ma, la isla pasa por un período de quiescencia y erosión. La Serie de las Cañadas se forma esencialmente entre los 1,9 Ma y los 0,2 Ma. La Serie dorsal se forma entre 0,8 a $0,5 \mathrm{Ma}$. Y por último, las Series recientes incluyen la actividad post-caldera del Complejo Teide-Pico Viejo y los ciclos históricos.

El modelo de crecimiento de la isla corresponde al menos a $5 \mathrm{Ma}$, con actividad discontinua y episodios de destrucción del relieve (Ancochea et al., op. cit.).

\section{La Gomera}

Han sido diferenciadas dos unidades geológicas: el Complejo Basal y las series volcánicas posteriores. La edad de los materiales subaéreos más antigua es de 10 Ma (Cantagrel et al., 1984). Han sido reconocidos varios ciclos volcánicos: uno entre los 9 y $6 \mathrm{Ma}$, otro entre los 4,5-4 Ma, y el último que tuvo lugar a los 2,8 Ma. Esta isla no ha tenido actividad volcánica durante el Cuaternario.

\section{La Palma}

En la Palma se han distinguido tres grandes unidades cronoestratigráficas: Complejo Basal, Series volcánicas antiguas y Series volcánicas recientes (Ancochea et al., 1994). La actividad subaérea se concentra en las dos últimas series, de las cuales la primera ocupa esencialmente la mitad norte de la isla y la segunda la mitad sur.

En las Series antiguas se distinguen dos unidades cronológicas principales: Serie Antigua I inferior datada entre 2 y 1,4 Ma; Serie Antigua I superior datada en 1 y 0,65 Ma.

En las Series recientes se distinguen dos unidades, una inferior -datada entre 0,6 a $0,15 \mathrm{Ma}-\mathrm{y}$ una unidad superior -posiblemente holocena (Ancochea et al., op. cit.).

\section{El Hierro}

Las formaciones geológicas existentes en la isla han sido agrupadas en tres series volcánicas (Fúster et al., 1993). En la Serie Antigua se pueden diferenciar dos períodos, uno con edades entre 0,8 a 0,7 Ma, y otro entre 0,4 y 0,2 Ma. Las Series Intermedia y Reciente tienen edades menores a $50 \mathrm{ka}$.

Las dos depresiones calderiformes de El Golfo y de Las Playas se han formado probablemente por grandes desplomes gravitatorios y avalanchas de edad bastante reciente, entre los 0,2 Ma y 6.000 a BP (Fúster et al., op. cit.).

\section{TIPOS DE YACIMIENTOS}

La mayor parte de los restos fósiles y subfósiles de vertebrados hallados en Canarias proceden de tubos volcánicos. Estos se localizan en el relleno sedimentario del tubo (p.e. Cueva del Llano, Fuerteventura) o bien, en superficie, en el suelo del mismo (p.e. Cueva del Viento, Tenerife). Huesos de grandes lacértidos, insectívoros, roedores y aves se han encontrado en este tipo de yacimiento. 
Otros materiales proceden de dunas fósiles, depósitos endorréicos, aluviales, tobas volcánicas, calcarenitas, y algunos se encuentran asociados a yacimientos arqueológicos como concheros, cuevas de enterramiento y habitación de los habitantes pre-europeos.

Las dunas pueden ser de dos tipos: las antiguas, que se encuentran cementadas por caliche y contienen abundantes nidos de himenópteros (Fuerteventura, Tenerife y La Graciosa), y las modernas que no están consolidadas (Corralejo, Fuerteventura). La alternancia de paleosuelos en estas dunas es utilizada en la reconstrucción paleoclimática (Meco, 1992; Meco y Petit-Maire, 1986). En las mismas han aparecido principalmente restos de lacértidos y aves marinas (Puffinus holeae).

Los derrubios de ladera y depósitos aluviales de fondo de valle están constituidos por sedimentos groseros y generalmente poco evolucionados. La importancia paleontológica de estos depósitos está limitada fundamentalmente por los procesos de remoción y por el tamaño de los sedimentos. La información paleontológica es puntual (Teno y San Andrés, Tenerife). En estos materiales se han hallado restos de Canariomys y Gallotia.

Otros depósitos que han suministrado importantes restos son las calcarenitas intercaladas entre coladas volcánicas. En estos materiales se encontraron huevos de "ratites" (Orzola, Lanzarote; Rothe, 1964) y huevos de tortugas terrestres de gran tamaño (Barranco de los Molinos, Fuerteventura; Rothe y Klemmer, 1991). Restos óseos de tortugas también han aparecido en tobas volcánicas (Adeje, Tenerife; Ahl, 1926).

\section{HISTORIA DE LOS HALLAZGOS PALEONTOLÓGICOS}

Lehrs (1909) atribuye a Lacerta simonyi un maxilar de un gran lacértido encontrado por Von Fritsch en 1873 en La Gomera. Esta es la primera cita de un vertebrado fósil en el Archipiélago, si bien a finales del siglo pasado fueron depositados en el Museo Canario de Las Palmas de Gran Canaria, unos restos de lacértidos incluidos en unos bloques de lapilli hallados en una cantera de La Isleta (Gran Canaria), pero estos permanecerían sin estudiar hasta hace pocos años (López Jurado, 1985).

Más tarde, Ahl (1926) describe una tortuga terrestre de gran tamaño (Testudo burchardi) encontrada en una cantera en Adeje (sur de Tenerife) (Burchard y Ahl, 1928; Burchard, 1934).

En 1939, el profesor J. Gómez de LLarena (San Sebastián) envió al Dr. Mertens unos restos procedentes de Tenerife, pero sin citar la localidad del hallazgo. Entre dichos materiales habían huesos de un lacértido de gran tamaño, de un roedor y de Puffinus. Los primeros fueron utilizados por Mertens para describir el primer lagarto fósil procedente de las Islas Canarias al que denominó Lacerta goliath (Mertens, 1942).

Posteriormente, Bravo (1953) designa una nueva especie de lacértido de gran tamaño, Lacerta maxima, procedente del acantilado de Martiánez (Puerto de la Cruz, Tenerife), la misma localidad tipo de L. goliath. En Bravo (1954) se realiza una ampliación de la distribución de L. maxima.

En 1958, al construirse un estanque en Jinamar (Gran Canaria) aparecen huevos pertenecientes a una tortuga terres- tre (Macau Vilar, 1960), que no fueron atribuidos a ninguna especie. En 1991, Rothe y Klemmer asignan estos restos a Testudo (Geochelone) burchardi.

En 1964, es descrito por Crusafont-Pairó y Petter el primer gran múrido del Archipiélago: Canariomys bravoi.

Rothe (1964) describe unos huevos de aves de gran tamaño y restos de cáscaras de los mismos, encontrados en Famara (norte de Lanzarote). Este investigador los atribuyó a las Ratites. Existían dos tipos, uno correspondiente al género Struthio (avestruces) y el otro a aves emparentadas con Aepyornis (ave elefante de Madagascar). Este hallazgo daría lugar a especulaciones sobre el origen continental de las Islas Canarias (Rothe y Schmincke, 1968; Sauer, 1972; Sauer y Rothe, 1972; Rothe, 1974).

Seguirían una serie de trabajos sobre las especies ya conocidas, profundizando en su descripción (Martínez Méndez, 1966; Gasc, 1971; García Cruz, 1978) o ampliando su distribución (Acosta Martínez y Pellicer Catalán, 1976; García Cruz y Marrero Rodríguez, 1979; Martín Esquivel, 1982).

En 1981, se citan restos de Gallotia simonyi en un yacimiento arqueológico de la isla de El Hierro (Böhme et al., 1981). Hasta ese momento se pensaba que el lagarto gigante sólo había habitado en los Roques de Salmor, donde se había extinguido en este siglo, y en Tenerife, donde se habían encontrado sus restos fósiles.

Hutterer (1985) describe nuevas subespecies (Gallotia goliath bravoana y Gallotia simonyi gomerana) para los lacértidos ya descritos, citando Gallotia goliath por primera vez para la isla de La Gomera.

López Martínez y López Jurado (1987) describen Canariomys tamarani, una rata de gran tamaño encontrada en La Aldea (Gran Canaria). Ese mismo año, en Lanzarote y Fuerteventura, se produce el hallazgo de los primeros restos fósiles de un insectívoro, la musaraña Crocidura canariensis, que aún habita en dichas islas (Hutterer et al., 1987). También se citó una nueva ave fósil, Carduelis triasi, para la isla de La Palma (Alcover i Florit, 1987). Por otra parte, Hirsch y López Jurado (1987) realizan un estudio de los huevos de tortugas terrestres aparecidos en Gran Canaria asignándolos al Plioceno.

Hutterer et al. (1988) describen un roedor, Malpaisomys insularis, de Lanzarote y Fuerteventura. Carrascosa y LópezMartínez (1988) citan a Mus musculus en la Cueva de Villaverde (Fuerteventura), mencionando que estos especímenes difieren de las subespecies descritas para Europa y Norte de Africa.

Izquierdo et al. (1989) amplían la distribución de Gallotia goliath a la isla de El Hierro. García-Talavera et al. (1989) realizan una recopilación de los yacimientos paleontológicos conocidos hasta ese momento en las canarias occidentales.

En 1990, se describen dos nuevas especies de pardelas fósiles: Puffinus holeae (Walker et al., 1990) y Puffinus olsoni (McMinn et al., 1990). Ambas especies fueron encontradas en Lanzarote y Fuerteventura. Ese mismo año, GarcíaTalavera atribuye al orden Odontopteryformes (aves marinas voladoras de gran envergadura) los restos determinados por Rothe como pertenecientes a Ratites del Mioceno de Lanzarote (García-Talavera, 1990).

Rothe \& Klemmer (1991) citan el hallazgo de huevos de tortugas fósiles en las calcarenitas de Fuerteventura, 
ampliando a esta isla la distribución de los quelonios terrestres hallados hasta ese momento.

Boye et al. (1992) realizan la reconstrucción de Malpaisomys insularis. Hutterer et al. (1992) hacen un estudio sobre el origen y evolución de las musarañas endémicas canarias.

En 1993 se realiza la descripción de una nueva especie de ave, Coturnix gomerae, hallada en una sima de la isla de La Gomera, junto a restos de otras aves y lacértidos (Jaume et al., en prensa).

Rando y Castillo (1993) describen un nuevo yacimiento de vertebrados en Fuerteventura (ramal de la Cueva del Llano, La Oliva).

El último fósil descrito para Canarias corresponde a la tortuga terrestre Geochelone vulcanica, un quelonio de gran tamaño del cual sólo se ha encontrado un resto (López Jurado y Mateo, 1993).

Castillo et al. (1994) estudian los restos momificados de dos lacértidos de gran tamaño en Tenerife y que corresponden a la especie extinguida Gallotia goliath.

\section{VERTEBRADOS FÓSILES}

La fauna fósil y subfósil de vertebrados de Canarias a la que nos referimos en este trabajo incluye reptiles, aves y mamíferos; los restos de peces no se han incluido pues apenas han sido citados en la bibliografía. La distribución geográfica de los yacimientos donde se han hallado los restos de estos grupos pertenecientes al Mio-Plioceno están representados en la Fig. 1; los del Pleistoceno y Holoceno se pueden observar en las Figs. 2 y 3, respectivamente.

\section{Reptiles}

a) Quelonios:

Testudo (Geochelone) burchardi

Geochelone vulcanica
Se trata de tortugas terrestres endémicas extinguidas, de gran tamaño, que poblaron algunas islas del archipiélago canario, al igual que ocurre actualmente en otras islas oceánicas y continentales como Galápagos y Seychelles.

Distribución: Testudo burchardi habitó en las islas de Tenerife y Fuerteventura. Geochelone vulcanica ha sido hallada en Gran Canaria (Tabla 1).

Datación: $T$. burchardi de Tenerife fue encontrada en materiales del Mioceno. Los restos de Fuerteventura se han datado en el Mio-Plioceno. Estas tortugas pudieron habitar en el Archipiélago hasta el Pleistoceno superior, ya que los restos hallados en Adeje (Tenerife) se encuentran en un estrato de toba volcánica que podría corresponder a este período (García-Talavera, com. pers.).

Relaciones filogenéticas: A pesar de no existir un registro fósil abundante, Rothe y Klemmer (1991) reconocen una estrecha relación entre T. (Geochelone) burchardi y el grupo de tortugas gigantes africanas Testudo (Geochelone) pardalis y Testudo (Geochelone) sulcata, que habitan la sabana africana en la actualidad. López Jurado y Mateo (1993) opinan que Testudo burchardi de Tenerife debe pasar a denominarse Geochelone burchardi.

Extinción: La desaparición de las tortugas terrestres gigantes pudo ser causada por los eventos volcánicos, al menos en el caso de Tenerife.

b) Lacértidos:

Género Gallotia Arnold, 1973

Gallotia goliath (extinguida)

Gallotia maxima (extinguida)

Gallotia simonyi

Gallotia stehlini

Gallotia galloti

Gallotia atlantica

Los lagartos del género Gallotia (Tabla 1), constituyen un grupo endémico de las Islas Canarias. Estos lacértidos presentan una tendencia al gigantismo tanto en el registro fósil

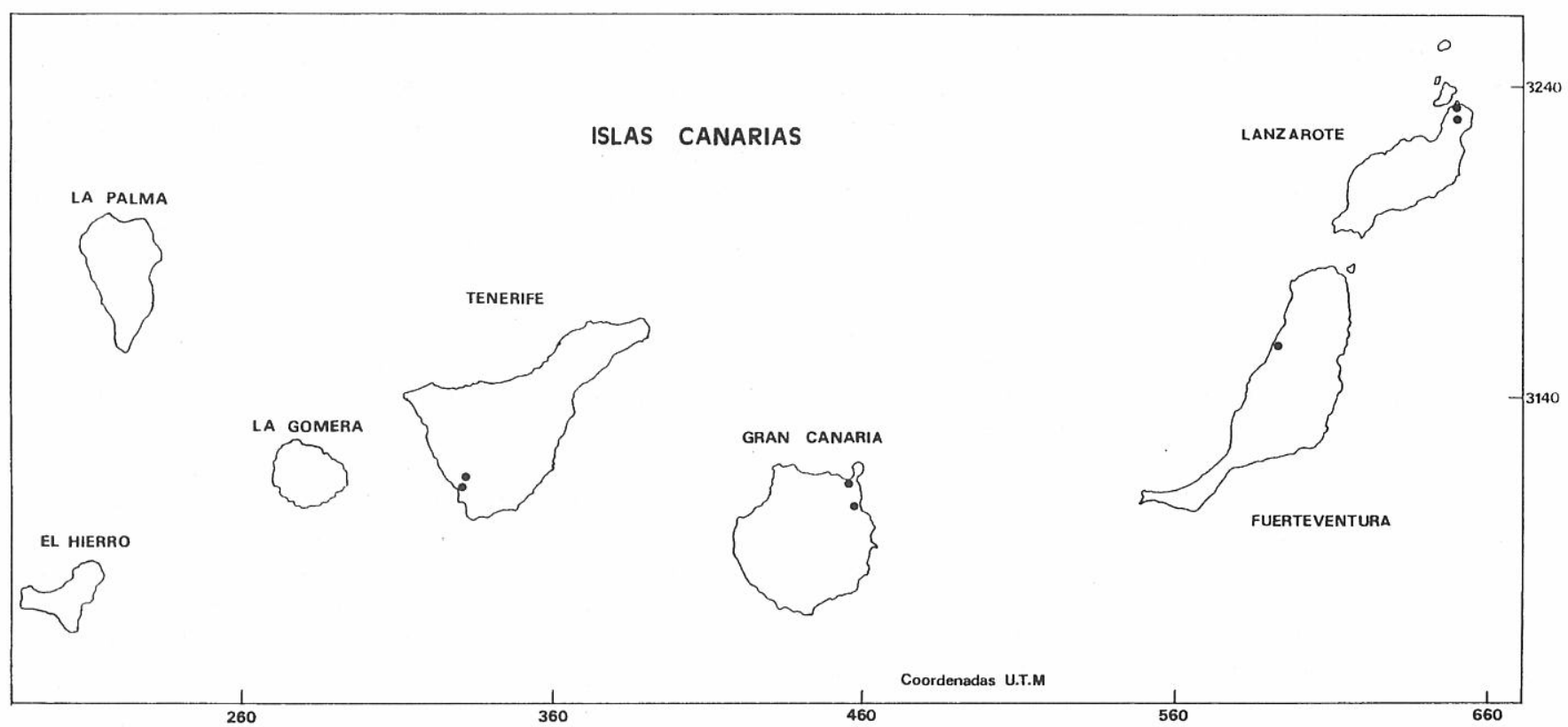

Figura 1. Localización geográfica de los yacimientos de vertebrados del Mioceno en el archipielago canario. 


\begin{tabular}{||l|l|l|l|l|l|l|l||}
\hline & L & F & C & T & P & G & H \\
\hline Testudo burchardi & & \# & $?$ & H & & & \\
\hline Geochelone vulcanica & & & $H$ & & & & \\
\hline Gallotia goliath goliath & & & & + & $*$ & & $*$ \\
\hline G. goliath bravoana & & & & & & $*$ & \\
\hline Gallotia maxima & & & & + & $*$ & & \\
\hline Gallotia simonyi simonyi & & & & $*$ & & & $*$ \\
\hline G. simonyi machadoi & & & & & & & $*$ \\
\hline G. simonyi gomerana & & & & & & $*$ & \\
\hline Gallotia galloti & & & & $*$ & & $*$ & $*$ \\
\hline Gallotia atlantica & $*$ & $*$ & & & & & \\
\hline Gallotia c. stehlini & & & $*$ & & & & \\
\hline Chalcides viridanus & & & & & & & $*$ \\
\hline Chalcides occidentalis & & $*$ & & & & & \\
\hline Chalcides sp. & & & $*$ & $*$ & & & \\
\hline Tarentola angustimentalis & & $*$ & & & & & \\
\hline T. boettgeri hierrensis & & & & & & & $*$ \\
\hline Tarentola sp. & $*$ & & $*$ & $*$ & & & \\
\hline
\end{tabular}

Tabla 1. Distribución de los reptiles fósiles y subfósiles de las Islas Canarias. Se han incluido las especies halladas en yacimientos arqueológicos (\#: Mio-Plioceno; +: Pleistoceno; *: Holoceno). ( $\mathrm{L}=$ Lanzarote, $\mathrm{F}=$ Fuerteventura, $\mathrm{C}=\mathrm{Gran}$ Canaria, $\mathrm{T}=$ Tenerife, $\mathrm{P}=\mathrm{La}$ Palma, $\mathrm{G}=\mathrm{La}$ Gomera, $\mathrm{H}=\mathrm{El}$ Hierro.

como en las poblaciones actuales. Este fenómeno se observa actualmente en especies de pequeño tamaño que se encuentran en condiciones de máximo aislamiento y su área habitable es pequeña (Martín, 1985; López Jurado y Mateo, 1992).

b.1) Gallotia goliath y Gallotia maxima

Estos lacértidos sólo se conocen en base a sus características osteológicas fragmentarias (Mertens, 1942; Bravo, 1953; Hutterer, 1985). El hallazgo de dos ejemplares de lagartos gigantes momificados en la isla de Tenerife-Barranco de las Moraditas- ha permitido, por primera vez, la descripción de las características morfológicas externas de la especie extinguida Gallotia goliath (Castillo et al. 1994). Los especímenes momificados son de gran tamaño, de cabeza plana, mejillas ensanchadas y hocico afilado. El número y la forma de los dientes premaxilares, maxilares y dentarios de los ejemplares momificados coinciden con los descritos para Gallotia goliath (Mertens, 1942; Hutterer, 1985).

Distribución: Gallotia goliath se encuentra en las islas occidentales del Archipiélago (Tabla 1), si bien la subespecie G. goliath bravoana sólo ha sido citada para La Gomera (Hutterer, op . cit.). Gallotia maxima se ha hallado en Tenerife y La Palma (Bravo, 1953; García Cruz y Marrero Rodríguez, 1979).

Datación: Los lagartos gigantes extinguidos han sido hallados en yacimientos correspondientes al Pleistoceno superior y al Holoceno (Tabla 1).

Relaciones filogenéticas: El análisis de las relaciones a nivel morfológico y osteológico de $G$. goliath con los restantes lacértidos del archipiélago, puso de manifiesto la estrecha similitud entre esta especie y $G$. simonyi.

Extinción: La extinción de $G$. goliath probablemente se debe a la acción de la colonización del hombre y al efecto de las relaciones de competencia con las poblaciones de la especie de pequeño tamaño (G. galloti) (Castillo et al., 1994).

\section{b.2) Gallotia simonyi}

Especie endémica de gran tamaño que se halla tanto en el registro fósil como en la actualidad.

Se han descrito tres subespecies: $G$. simonyi simonyi y G. simonyi gomerana, extinguidas; G. simonyi machadoi, amenazada de extinción actualmente.

Distribución: Los restos fósiles han sido hallados en Tenerife, La Gomera y El Hierro (Tabla 1).

Datación: Se han encontrado desde el Pleistoceno superior (Bravo, 1953) hasta la actualidad.

\section{b.3) Gallotia stehlini}

Lacértido endémico de gran tamaño. Se diferencia de las otras especies de lagartos gigantes ( $G$. goliath, G. maxima, $G$. simonyi) por el número de cúspides dentarias y morfología del parietal (Hutterer, 1985).

Distribución: Gran Canaria.

Datación: Cuaternario.

b.4) Gallotia galloti y Gallotia atlantica.

Especies endémicas de pequeño tamaño. G. galloti ha sufrido un proceso de evolución insular dando lugar a varias subespecies.

Distribución: Los restos fósiles de G. galloti se han hallado en Tenerife, La Gomera y El Hierro. G. atlantica se ha encontrado en Lanzarote y Fuerteventura.

Datación: En ambos casos aparecen desde el Pleistoceno hasta la actualidad.

Observaciones: Algunos investigadores piensan que los dos lagartos de gran tamaño descritos (Gallotia goliath y Gallotia maxima) constituyen una única especie. Las investigaciones que se realizan en la actualidad intentan dilucidar el estatus taxonómico de estos reptiles.

Las diferencias de tamaño observadas en los ejemplares momificados de Gallotia goliath también existen entre las formas fósiles y formas actuales de Gallotia stehlini en Gran Canaria (Mateo y López Jurado, 1992), de G. simonyi en la isla de El Hierro (Izquierdo et. al., 1989); y en las poblaciones actuales de G. galloti en Tenerife (Martín, 1985), La Palma, La Gomera y El Hierro (Bischoff, 1982). Estas mismas variaciones han sido observadas en $G$. atlantica, en la isla de Lanzarote (López Jurado y Mateo, 1992). Existe, por tanto, una tendencia hacia el gigantismo en Gallotia, cuando el área habitada es pequeña, como ocurrió en el pasado en los islotes de Roque Chico de Salmor (G. simonyi simonyi), y en el presente en Roque Fuera de Anaga (G. galloti insulanagae; Martín, 1985) y distintos ecosistemas ("malpaís" e "islotes") de la isla de Lanzarote (G. atlantica; López Jurado y Mateo, 1992).

\section{Aves}

a) Grandes aves extinguidas:

Como ya se ha mencionado anteriormente los huevos de estas aves han sido atribuidos al género Struthio (avestruces actuales) y a aves emparentadas con Aepyornis (Rothe, 1964; Sauer y Rothe, 1972) y al orden Odontopteryformes (GarcíaTalavera, 1990).

Distribución: Norte de la isla de Lanzarote.

Datación: Mioceno (Rothe, 1964). García-Talavera (op. cit.) les atribuye una edad de 6 millones de años.

b) Otras especies de aves endémicas de Canarias extinguidas: Coturnix gomerae, Carduelis triasi, Puffinus holeae y P. olsoni (Tabla 2). 


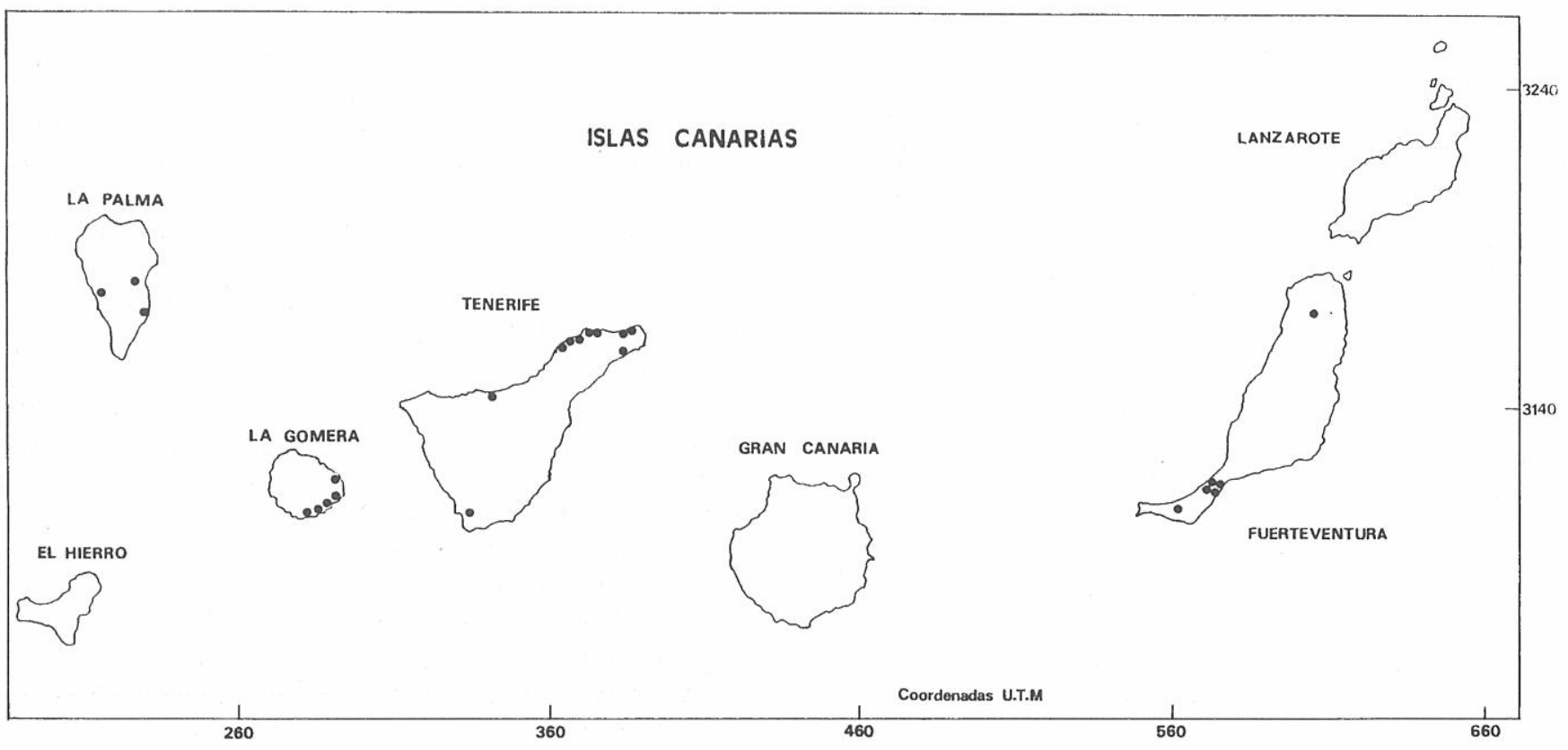

Figura 2. Localización geográfica de los yacimientos de vertebrados del Pleistoceno en el archipiélago canario.

\section{b.1) Carduelis triasi}

Paseriforme eminentemente terrestre y con una capacidad voladora mermada. Posee una mayor talla y las alas son más pequeñas que las de $C$. chloris (Verderón).

Distribución: Ha sido hallado en la isla de La Palma.

Datación: Pleistoceno superior-Holoceno reciente.

Extinción: Su desaparición fue posiblemente coetánea con la presencia humana en la isla (Alcover y Florit, 1987).

\section{b.2) Puffinus holeae}

Esta pardela poseía una talla mayor que $P$. puffinus (la mayor especie de este género, nidificante en Canarias) y construía madrigueras para nidificar en dunas de arena consolidadas, donde se encuentran actualmente sus restos en grandes cantidades (Walker et al., 1990).

Distribución: Lanzarote y Fuerteventura

Datación: Pleistoceno superior y Holoceno.

b.3) Puffinus olsoni

Procelariforme que poseía una talla intermedia entre $P$. puffinus y $P$. assimilis. De hábitos cavernícolas (McMinn et al., 1990), los restos se hallan principalmente en zonas de "malpaís".

Distribución: Lanzarote, Fuerteventura y La Graciosa.

Datación: Holoceno reciente

Extinción: Puffinus holeae y P. olsoni parecen haber desaparecido en época reciente, posteriormente a la colonización humana de las islas (Walker et al., 1990; McMinn et al., 1990). Restos de P. olsoni se han hallado en niveles superficiales de yacimientos arqueológicos de Fuerteventura, con claras evidencias de haber sido consumidas por los aborígenes de esta isla (Rando y Perera, 1994).

b.4) Coturnix gomerae

Los huesos de las extremidades de esta codorniz indican que poseía hábitos más terrestres y menos voladores que $C$. coturnix (Jaume et al., en prensa).

Distribución: La Gomera (Jaume et al., en prensa) y Fuerteventura (Rando y Perera, 1994).
Datación: Holoceno

Extinción: Según Rando y Perera (op. cit.) es posterior a la llegada del hombre al archipiélago, ya que sus restos han aparecido en un yacimiento arqueológico de Fuerteventura.

Observaciones: La aparición de restos de esta ave en más de una isla podría poner de manifiesto una radiación de este género en Canarias (Alcover i Florit, 1989) al igual que ocurrió en Madeira y Porto Santo (Pieper, 1985).

b.5) Otros restos de aves hallados en los yacimientos canarios están recogidos en la tabla 2. Entre ellos podemos destacar:

- Los restos óseos pertecientes a pigargo (Haliaetus sp.) hallados en yacimientos paleontológicos de Fuerteventura (Rando, en prep.) y recientemente por nosotros en la isla de El Hierro. Esta ave de presa podría representar la cima de la pirámide ecológica durante el Cuaternario superior en Canarias (Rando, en prep.).

- La presencia de palomas, íntimamente relacionadas con las palomas actuales de los bosques de laurisilva de Canarias (Columba bollii y C. junoniae) en Fuerteventura (Alcover, com. pers.) y en Lanzarote. Su hallazgo en los yacimientos arqueológicos de las islas más áridas del archipiélago contrastaría con sus hábitats actuales.

\section{Mamíferos}

Dentro de este grupo están representados los roedores, insectívoros, lagomorfos, quirópteros, félidos, cánidos, ovicáprinos, suidos, cetáceos y fócidos (Tabla 3).

a) Roedores:

a.1) Canariomys bravoi y Canariomys tamarani

Son especies endémicas, que presentan gigantismo, y que están extinguidas en el Archipiélago canario. Canariomys bravoi se caracteriza por tener convergencias con especies marchadoras y cavadoras (Espresate, 1991). Cana- 


\begin{tabular}{|c|c|c|c|c|c|c|c|}
\hline & $\mathrm{L}$ & $\mathrm{F}$ & $\mathrm{C}$ & $\mathrm{T}$ & $P$ & G & $\mathrm{H}$ \\
\hline "Grandes aves" & $\#$ & & & & & & \\
\hline Diomedea sp. & & & & $*$ & & & \\
\hline cf. Puffinus assimilis & & & $*$ & & & & \\
\hline Puffinus holeae & + & + & & & & & \\
\hline Puffinus olsoni & $*$ & $*$ & & & & & \\
\hline Calonectris diomedea & $*$ & * & & & $*$ & & $*$ \\
\hline Haliaetus sp. & & $?$ & & & & & \\
\hline Accipiter nisus & & & * & & & & \\
\hline Buteo buteo & & & * & & & & $*$ \\
\hline Buteo buteo insularum & & & & & & * & \\
\hline Neophron percnopterus & & & & & & $*$ & \\
\hline Falco tinnunculus & & & * & & $*$ & $*$ & \\
\hline Coturnix gomerae & & $?$ & & & & * & * \\
\hline Coturnix sp. & & & $*$ & & & & \\
\hline Burhinus oedicnemus & & & * & & & ' & \\
\hline Charadrius sp. & & & * & & & & \\
\hline Larus cachinans & & & & & & & $*$ \\
\hline Columba junoniae/bollii & $*$ & $*$ & $*$ & & & $*$ & $*$ \\
\hline Columba sp. & & $*$ & * & * & & $*$ & \\
\hline Tyto cf. alba & $*$ & & & & & & \\
\hline Tyto alba gracilirostris & & & & & & * & \\
\hline Tyto sp. & & & $*$ & & & & \\
\hline Asio otus & $*$ & & & & $*$ & & \\
\hline Anthus berthelotii & ${ }^{*}$ & $*$ & & & & & \\
\hline Turdus merula & & & $*$ & & & & \\
\hline Turdus sp. & ${ }^{*}$ & $*$ & * & & & & \\
\hline Sylvia cf. atricapilla & & $*$ & & & & & \\
\hline Phyrrocorax phyrrocorax & & & & * & $*$ & $*$ & $*$ \\
\hline Corvus corax & * & & $*$ & & & $*$ & $*$ \\
\hline Corvidae & ${ }^{*}$ & & & & & & \\
\hline Carduelis triasi & & & & & $*$ & & \\
\hline Carduelis chloris & & & & & & $*$ & \\
\hline
\end{tabular}

Tabla 2. Distribución de las aves fósiles y subfósiles de las Islas Canarias. Se han incluido las especies halladas en yacimientos arqueológicos (\#: Mio-Plioceno; +: Pleistoceno; *: Holoceno). ( $\mathrm{L}=$ Lanzarote, $\mathrm{F}=$ Fuerteventura, $\mathrm{C}=\mathrm{Gran}$ Canaria, $\mathrm{T}=$ Tenerife, $\mathrm{P}=\mathrm{La}$ Palma, $\mathrm{G}=\mathrm{La}$ Gomera, $\mathrm{H}=\mathrm{El}$ Hierro).

riomys tamarani es un roedor de hábitos vegetarianos y cavadores (López-Martínez y López-Jurado, 1987).

Distribución: Canariomys bravoi ha sido hallada en Tenerife y C. tamarani en Gran Canaria.

Datación: Canariomys bravoi ha aparecido en depósitos pleistocenos y holocenos; los restos de C. tamarani están ligados a yacimientos holocenos (López-Martínez y LópezJurado, op. cit.).

a.2) Malpaisomys insularis

El estudio del esqueleto, la reconstrucción del tamaño, forma y nicho ecológico de Malpaisomys ha sido realizado por Boye et al. (1992). Según estos autores, se trata de una especie con adaptaciones en los miembros anteriores y posteriores para trepar, apto para su nicho ecológico inferido, el malpaís.

Distribución: Ha sido hallado en diversos yacimientos de Fuerteventura, Lanzarote y La Graciosa (Hutterer et al., 1988; Rando y Castillo, 1993).

Datación: Esta especie fue descrita en el yacimiento arqueológico de la Cueva de Villaverde (La Oliva, Fuerte- ventura) que está datado entre los 1000 y 1700 a BP (Hutterer et al., 1988). Su presencia en el Pleistoceno superior es señalada por Michaux et al. (1991).

Relaciones filogenéticas: Los estudios sobre su filogenia, basados en las características morfológicas, han sido llevados a cabo por Hutterer et al. (1988), que lo relacionan con Acomys y Uranomys. El análisis de las albúminas fósiles de Malpaisomys ha dado como resultado el establecimiento de otra filogenia diferente, estando éste más emparentado con el género Mus (Montgelard, 1991).

Extinción: Según Boye et al. (1992) se pudo deber, entre otros factores, a su desplazamiento por la llegada de Mus musculus o bien a cambios ambientales. Estos cambios en el medio ambiente se reflejan en la disminución de la talla de Mus musculus en los últimos 2.000 años (Carrascosa y López-Martínez, 1988).

a.3) Mus musculus

Esta especie, generalmente ligada a yacimientos arqueológicos, presenta unas características osteológicas y dentarias particulares en la población procedente de la Cueva de Villaverde, lo que ha llevado a Carrascosa y López-Martínez (op.cit.) a proponer la adscripción de dicho material a una nueva subespecie.

Distribución: La especie vive en la actualidad en todas las islas del Archipiélago. Restos subfósiles se encuentran en Gran Canaria (Boye et al., 1992), El Hierro (Castillo et al., en prep.) y Fuerteventura (Carrascosa y López-Martínez, 1988; Rando y Castillo, 1993).

Datación: La entrada en el archipiélago de esta especie, se hace coetánea con la llegada del hombre, aunque no se descarta una colonización anterior (Carrascosa y López Martínez, op. cit.).

\begin{tabular}{||l|c|c|c|c|c|c|c||}
\hline & $\mathrm{L}$ & $\mathrm{F}$ & $\mathrm{C}$ & $\mathrm{T}$ & $\mathrm{P}$ & $\mathrm{G}$ & $\mathrm{H}$ \\
\hline Malpaisomys insularis & $+{ }^{*}$ & $+^{*}$ & & & & & \\
\hline Canarionys bravoi & & & & $+*$ & & & \\
\hline Canariomys tamarani & & & $*$ & & & & \\
\hline Rattus sp. & $*$ & & & $*$ & & & $*$ \\
\hline Mus musculus & & & $*$ & & & & $*$ \\
\hline Mus musculus ssp. & & $*$ & & & & & \\
\hline Crocidura canariensis & $+*$ & $+*$ & & & & & \\
\hline Oryctolagus cuniculus & $*$ & & $*$ & $*$ & & & $*$ \\
\hline Quirópteros & & & & $*$ & & & $*$ \\
\hline Capra hircus & $*$ & $*$ & $*$ & $*$ & & $*$ & $*$ \\
\hline Ovis aries & $*$ & & & $*$ & & $*$ & $*$ \\
\hline Ovis cf. aries & & $*$ & & & & & $*$ \\
\hline Ovicápridos & & & & & $*$ & & \\
\hline Sus scrofa & & $*$ & & & & $*$ & \\
\hline Sus scrofa porcus & $*$ & & $*$ & $*$ & & & $*$ \\
\hline Sus domesticus & & & & & & $*$ & \\
\hline Suidos & & & & & $*$ & & \\
\hline Canis familiaris & $*$ & & $*$ & $*$ & $*$ & $*$ & $*$ \\
\hline Felis catus & & & & $*$ & $*$ & & $*$ \\
\hline Monachus monachus & & $*$ & & & & & \\
\hline Cetáceos & $*$ & & $*$ & & $*$ & & $*$ \\
\hline
\end{tabular}

Tabla 3. Distribución de los mamíferos fósiles y subfósiles de las Islas Canarias. Se han incluido las especies halladas en yacimientos arqueológicos. (+: Pleistoceno; *: Holoceno). ( $\mathrm{L}=$ Lanzarote, $\mathrm{F}=$ Fuerteventura, $\mathrm{C}=$ Gran Canaria, $\mathrm{T}=$ Tenerife, $\mathrm{P}=\mathrm{La}$ Palma, $\mathrm{G}=\mathrm{La}$ Gomera, $\mathrm{H}=\mathrm{El}$ Hierro). 


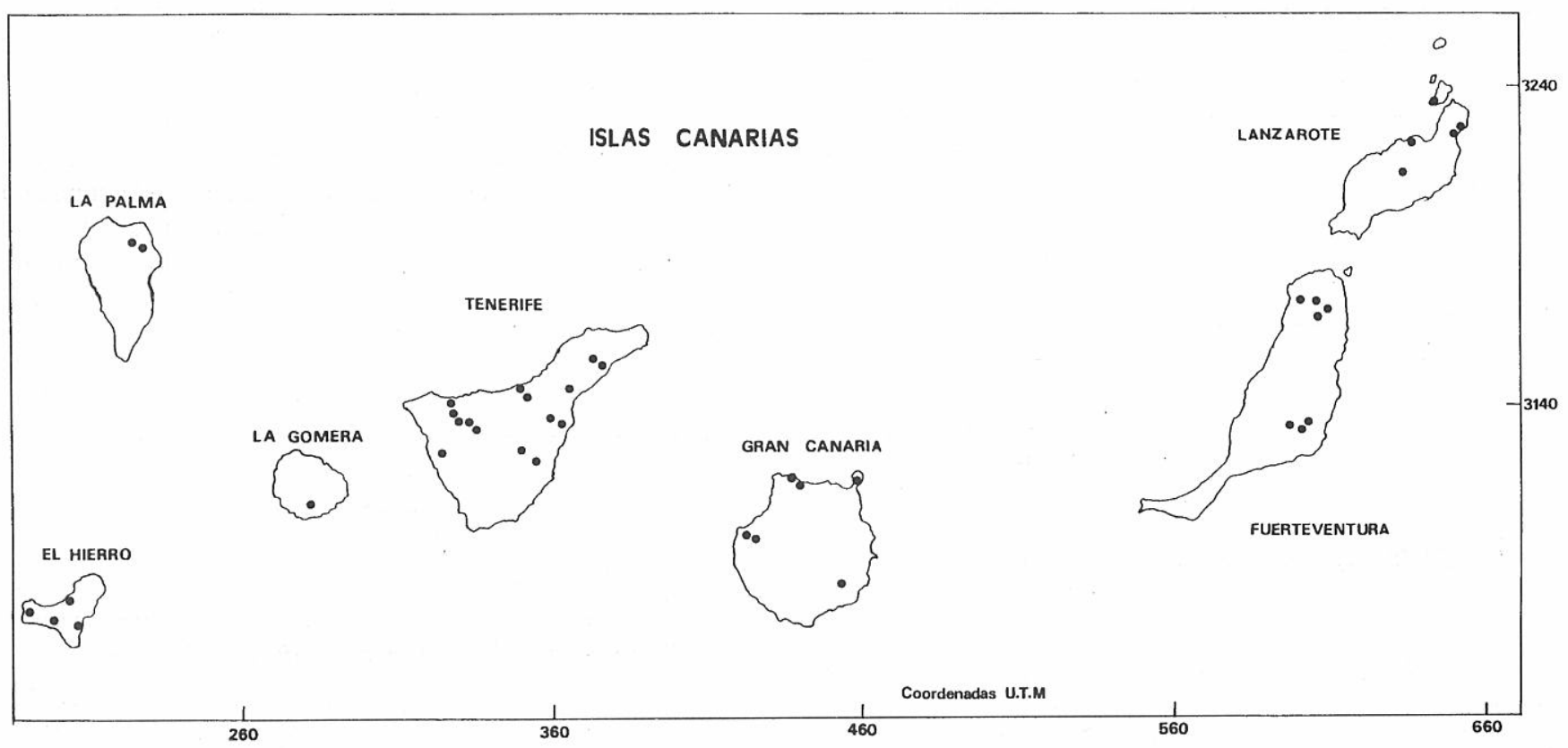

Figura 3. Localización geográfica de los yacimientos de vertebrados del Holoceno en el archipiélago canario.

\section{b) Insectívoros}

Los restos fósiles y subfósiles hallados hasta el momento en Canarias se adscriben a la especie endémica Crocidura canariensis (Tabla 3). Los ejemplares fósiles de esta especie presentan un mayor tamaño que los especímenes actuales.

Distribución: Lanzarote y Fuerteventura

Datación: Pleistoceno superior (Michaux et al., 1991) hasta la actualidad.

Relaciones filogenéticas: Según Hutterer et al. (1992), después de haber realizado el análisis genético ( $\mathrm{n}^{\circ}$ de cromosomas y distancia genética), análisis de la vocalización y el estudio morfológico, $C$. canariensis está relacionada filogenéticamente con $C$. sicula y no está emparentada con $C$ rocidura osorio, la otra musaraña que habita actualmente en las islas.

c) Otros mamíferos:

En la Tabla 3 se citan las especies correspondientes a lagomorfos, félidos, cánidos, ovicáprinos, suidos, cetáceos y fócidos hallados hasta el momento en Canarias. Estas especies están ligadas a yacimientos arqueológicos y su estudio está dirigido a conocer el grado de asociación con los hábitos culturales del hombre.

Los restos de quirópteros son poco conocidos. Se encuentran citados en el yacimiento de Martiánez (Puerto de la Cruz, Tenerife; Bravo, 1953; Martínez Méndez, 1966) y en el yacimiento arqueológico de la Cueva de la Enladrillada (Tegueste, Tenerife; Diego Cuscoy, 1972). Recientemente, en el curso de nuestras investigaciones los hemos hallado en tubos volcánicos de la isla de El Hierro.

\section{LEGISLACIÓN VIGENTE}

En 1982 se aprueba el Estatuto de Autonomía de Canarias, recogida en la Ley Orgánica 10/1982 de 10 de Agosto. Esta ley en el artículo 29.9 atribuye a la Comunidad Autó- noma competencias exclusivas en materia de Patrimonio Histórico.

La Ley 16/1985, de 25 de Junio, del Patrimonio Histórico Español obliga (Título V, Art. 42) a que cualquier excavación o prospección (histórica o paleontológica) (Art. 41) relacionada con la historia del hombre y sus orígenes y antecedentes (Art. 40) debe ser autorizada por la Administración competente, que en el caso de Canarias es en la actualidad la Dirección General de Patrimonio de la Viceconsejería de Cultura y Deportes del Gobierno de Canarias, y en el pasado fue la Dirección General de Cultura de la misma. En este apartado hay que incluir a la mayoría de los yacimientos con restos fósiles de vertebrados de Canarias, pues aparecen en yacimientos arqueológicos bien junto a la fauna doméstica de los mismos o en los niveles inferiores de estos yacimientos (p.e. Cueva del Bco. de la Arena, Tenerife)

La Viceconsejería de Cultura y Deportes mediante una Orden de 5 de Febrero de 1987 regula las autorizaciones para la realización de investigaciones arqueológicas, paleontológicas y etnológicas. En ella se hace constar que en las solicitudes de excavaciones paleontológicas se debe contar con la colaboración de un arqueólogo, exigiendo además que éste tenga experiencia en este tipo de actividades. Asimismo, según el artículo 42.2 de la Ley del Patrimonio Histórico Español los materiales procedentes de las excavaciones arqueológicas (en el que están incluidos los elementos geológicos y paleontológicos relacionados con el hombre) deben ser depositados en el museo o centro que la Administración competente designe (generalmente Museos Arqueológicos).

En 1991 se suspenden en Canarias las autorizaciones para la realización de Investigaciones Arqueológicas, Paleontológicas y Etnológicas (Orden de 29 de Octubre de 1991), debido a los desiguales resultados, no siempre positivos, obtenidos hasta ese momento en las autorizaciones de proyectos y campañas de excavaciones concedidas. Tras dos años de suspensión se vuelve a convocar el concurso de con- 
cesión, siendo la Comisión Regional de Patrimonio Histórico de Canarias quien valore y seleccione las solicitudes. En esta Orden no se menciona la necesidad de contar con un arqueólogo para las excavaciones y prospecciones paleontológicas, pero este trámite se ha continuado exigiendo en las sucesivas convocatorias realizadas.

En la actualidad los Cabildos tienen las competencias en materia de vigilancia y conservación de los yacimientos, pero las autorizaciones para investigaciones y excavaciones las tiene la Dirección General de Patrimonio. Los Cabildos están reclamando mayores competencias en materia de Patrimonio y una nueva ley está próxima a entrar en el Parlamento de Canarias, cuyo contenido se desconoce por el momento.

\section{ESTADO DE CONSERVACIÓN DE LOS YACIMIENTOS}

Los yacimientos canarios se encuentran amenazados por la construcción de urbanizaciones turísticas, por su saqueo, por las filtraciones de aguas de riego y fecales procedente de la superficie en el caso de los tubos volcánicos, las extracciones de picón o arena para las construcciones, entre otros factores.

La única protección existente hasta el momento proviene de la ley de Espacios Naturales de Canarias, aprobada el 16 de Noviembre de 1994, en la cual la existencia de yacimientos paleontológicos de interés científico es uno de los aspectos que se considera, solo o unido a otros factores, para la declaración de una zona como espacio natural protegido (Título III, Cap. 1, Art. 8). En esta misma ley se contempla la figura de Monumento Natural en el que se incluyen las formaciones geológicas y yacimientos paleontológicos que reunan un interés especial por su singularidad o importancia y por sus valores científicos (Título III, Cap. 1, Art. 12.1).

\section{CONCLUSIONES}

1. ${ }^{\circ}$ El conocimiento de la fauna fósil es puntual, haciendo falta trabajos de síntesis y prospecciones paleontológicas sistemáticas.

2. ${ }^{\circ}$ Se han descrito ocho especies de reptiles, cuatro especies de mamíferos y dos especies de aves endémicas y terrestres, y dos especies de aves marinas. Su distribución comprende desde el Mio-Plioceno al Holoceno. De ellas se han extinguido cuatro especies de reptiles, tres de mamíferos y al menos cinco especies de aves. En muchos casos la extinción pudo estar relacionada con el hombre.

3. ${ }^{\circ}$ El origen, colonización y radiación evolutiva de las distintas especies de vertebrados están actualmente en debate.

4. ${ }^{\circ}$ Es necesario hacer una revisión detallada del estatus taxonómico de los quelonios endémicos terrestres del Archipiélago y del género Gallotia, y caracterizar las especies fósiles y subfósiles de este último.

5. ${ }^{\circ}$ Los restos de aves están relacionados generalmente con la fauna de los yacimientos arqueológicos. Han habido importantes extinciones y cambios en la distribución geo- gráfica de ellas, como se puede apreciar en los últimos resultados obtenidos.

6. ${ }^{\circ}$ Es necesaria una nueva Ley de Patrimonio en la Comunidad Canaria que recoja las necesidades de los paleontólogos y la protección de los yacimientos, así como se precisa una mayor vigilancia para evitar el saqueo de estos últimos.

\section{AGRADECIMIENTOS}

Agradecemos al Dr. Jorge Morales y a la Dra. Nieves López-Martínez, la revisión crítica de este trabajo.

\section{BIBLIOGRAFÍA}

Acosta Martínez, P. y Pellicer Catalán, M. 1976. Excavaciones arqueológicas en la Cueva de la Arena (Barranco Hondo, Tenerife). Anuario de Estudios Atlánticos, 22, 125-184.

Ahl, E. 1926. Über eine ausgestorbene Riesenchildkröte der Insel Teneriffa. Zeitschrift der deutschen Geologischen Gessellschaft, 77, 575-580.

Alcover, J. A. y Florit, F. 1987. Una nueva especie de Carduelis (Fringillidae) de La Palma. Vieraea, 17, 75-86.

Alcover, J. A. i Florit, F. 1989. Els ocells del jaciment arqueològic de La Aldea, Gran Canaria. Butlleti Institut Catala Història Natural, 56, 47-55.

Ancochea, E., Fúster, J. M., Ibarrola, E., Cendrero, A., Coello, J., Hernán, F., Cantagrel, J. M. and Jamond, C. 1990. Volcanic evolution of the island of Tenerife (Canary Islands) in the light of new K-Ar data. Journal of Volcanology and Geothermal Research, 44, 231-249.

Ancochea, E., Hernán, F., Cendrero, A., Cantagrel, J. M., Fúster, J. M., Ibarrola, E. y Coello, J. 1994. Constructive and destructive episodes in the building of a young Oceanic Island, La Palma, Canary Islands, and genesis of the Caldera de Taburiente. Journal of Volcanology and Geothermal Research, 60 , 243-262.

Arnold, E. N. 1973. Relationships of the paleartic Lizards assigned to the genera Lacerta, Algyroides and Psammodromus (Reptilia, Lacertidae). Bulletin British Museum Natural History Zoology, 25, 289-366.

Bischoff, W. 1982. Die innerartliche Gliederung von Gallotia gallo$t i$ (DUMERIL \& BIBRON, 1839) (Reptilia: Sauria: Lacertidae) auf Teneriffa, Kanarische Inseln. Bonner Zoologische Beiträge, 33, 363-382.

Böhme, W., Bischoff, W., Nettmann, H. K., Rikena, S. and Freundlich, J. 1981. Nachweis von Gallotia simonyi (Steindachner, 1889) (Reptilia: Lacertidae) auf einer frümittelalterlichen Fundschcht auf Hierro, Kanarische Inseln. Bonner Zoologische Beiträge, 32, 157-166.

Boye, P., Hutterer, R., López-Martínez, N. and Michaux, J. 1992. A reconstruction of the Lava mouse (Malpaisomys insularis), an extinct rodent of the Canary Islands. Zeitschrift fur Säugetierkunde, 57, 29-38.

Bravo, T. 1953. Lacerta maxima $\mathrm{n}$. sp. de la fauna continental extinguida en el Pleistoceno de las Islas Canarias. Estudios Geológicos, 9, 7-34.

Bravo, T. 1954. Geografía General de las Islas Canarias. Tomo I. Ed Goya. Sta. Cruz de Tenerife. 
Burchard, O. 1934. Testudo burchardii, E. Ahl. El primer gran fósil descubierto en Canarias. Instituto Estudios Canarios, Monografía, Sec. IV, 1, 1-15.

Burchard, O. and Ahl, E. 1928. Neue Funde von Riesen-landschildkröten auf Teneriffa. Zeitschrift der deutschen Geologischen Gessellschaft, 79, 439-447.

Cantagrel, J. M., Cendrero, A., Fuster, J. M., Ibarrola, E. and Jamond, C. 1984. K-Ar Chronology of the volcanic Eruptions in the Canarian Archipelago: Island of La Gomera. Bulletin Volcanologique, 47-3, 597-609.

Carrascosa, M. C. and López-Martínez, N. 1988. The house mouse from a prehistoric site in Fuerteventura (Canary Islands, Spain). Bonner zoologische Beiträge, 39, 237-256.

Castillo, C., Rando, J. C. and Zamora, J. F. 1994. Discovery of mummified extinct giant lizards (Gallotia goliath, Lacertidae) in Tenerife, Canary Islands. Bonner zoologische Beiträge, 45, 2 .

Coello, J., Cantagrel, J. M., Hernán, F., Fúster J. M., Ibarrola, E., Ancochea, E., Casquet, C., Jamond, C., Díaz de Téran, J. R. and Cendrero, A. 1992. Evolution of the eastern volcanic ridge of the Canary Islands based on new K-Ar data. Journal of Volcanology and Geothermal Research, 53, 251-274.

Crusafont-Pairó, M et Petter, F. 1964. Un Murine géant fossile des Iles Canaries Canariomys bravoi gen. nov. sp. nov. (Rongeur, Muridés). Mammalia, 28, 608-611.

Diego Cuscoy, L. 1972. La Necropólis de "La Enladrillada" (Tegueste-Tenerife). En: Excavaciones Arqueológicas en Tegueste. Noticiario Arqueológico Hispánico, Prehistoria I, 271-313.

Espresate, J. 1991. Evolución insular de Canariomys bravoi (Crusafont y Petter 1964). Revista Española de Paleontología, n. ${ }^{\circ}$ extra, 47-51.

Fuster, J. M., Hernán, F., Cendrero, A, Coello, J, Cantagrel, J. M., Ancochea, E e Ibarrola, E. 1993. Geocronología de la Isla de El Hierro (Islas Canarias). Boletín Real Sociedad Historia Natural (Sección Geología), 88, 85-97.

García Cruz, C. M. 1978. Características anatómicas y biométricas de Lacerta maxima Bravo, 1953 (Reptilia: Lacertidae). Memoria de Licenciatura. Universidad de La Laguna, 1-192 (sin publicar).

García Cruz, C. M. y Marrero Rodríguez, A. 1979. Sobre la distribución geográfica de los yacimientos de vertebrados fósiles de las Islas Canarias. Vieraea, 8, 95-100.

García-Talavera, F. 1990. Aves gigantes en el Mioceno de Famara (Lanzarote). Revista Academia Canaria de las Ciencias, 2, 71-79.

García-Talavera Casañas, F., Paredes Gil, R. y Martín Oval, M. 1989. Catálogo-Inventario yacimientos paleontológicos. Provincia de Sta. Cruz de Tenerife. Instituto de Estudios Canarios, $1-76$.

Gasc, J. P. 1971. Les variations columnaires dans la région présacrée des sauriens. Application a la reconstitution de Lacerta goliath Mertens. Annales de Paléontologie (Vertébrés), LVII, 133-155.

Hernán Reguera, F. 1993-95. Entorno geológico y geodinámico y origen del Archipiélago Canario. Master en Gestión Ambiental. Universidad de La Laguna.

Hirsch, K. F. and López-Jurado, L. F. 1987. Pliocene chelonian eggs from Gran Canaria, Canary Islands. Journal of Vertebrate Paleontology, 7, 96-99.
Hutterer, R. 1985. Neue Funde von Rieseneidechsen (Lacertidae) auf der Insel Gomera. Bonner Zoologische Beiträge, 36, 365-394.

Hutterer, R., López-Jurado, L. F. and Vogel, P. 1987. The shrews of the eastern Canary Islands: a new species (Mammalia: Soricidae). Journal of Natural History, 21, 1347-1357.

Hutterer, R., López-Martínez, N. and Michaux, J. 1988. A new rodent from Quaternary deposits of the Canary Islands and its relationships with neogene and recent murids of Europe and Africa. Paleovertebrata, 18, 241-262.

Hutterer, R., Maddalena, T. and Molina, O. M. 1992. Origin and evolution of the endemic Canary Island shrews (Mammalia: Soricidae). Biological Journal of the Linnean Society, 46, 49-58.

Izquierdo, I., Medina, A. L. and Hernández, J. J. 1989. Bones of giant lacertids from a new site on El Hierro (Canary Islands). Amphibia-Reptilia, 1, 63-69.

Jaume, D., McMinn, M. and Alcover, J. A. (en prensa). The fossil birds from the Bujero del Silo, La Gomera (Canary Islands, Eastern Atlantic Ocean). Boletim do Museu Municipal do Funchal.

Lehrs, P. H. 1909. Studien über Abstammung und Ausbreitung in den Formenkreisen der Gattung Lacerta und ihrer Verwandten. Zoologische Jahrbuch Systematisch, 28, 1-38

López-Jurado, L. F. 1985. Los reptiles fósiles de la Isla de Gran Canaria (Islas Canarias). Bonner Zoologische Beiträge, 36, 355-364.

López-Martínez, N. y López-Jurado, L. F. 1987. Un nuevo múrido gigante del Cuaternario de Gran Canaria, Canariomys tamarani n. sp. (Mammalia, Rodentia). Doñana, Publ. Oc., 2, 1-60.

López-Jurado, L. F. and Mateo, J. A. 1992. Two models of evolution in Canarian lizards based on the use of spatial resources. Biological Journal of the Linnean Society 46, 25-37.

López-Jurado, L. F and Mateo, J. A. 1993. A new giant land tortoise from the Pliocene of Gran Canaria (Canary Islands). Revista Española de Herpetología, 7, 107-111.

Macau Vilar, F. 1960. Contribución al estudio del Cuaternario de Gran Canaria. Anuario de Estudios Atlánticos, 6, 117-132.

Martín, A. 1985. Los lagartos de los Roques del Norte de Tenerife. Bonner zoologische Beiträge, 36, 517-528.

Martín Esquivel, J. L. 1982. El ecosistema cavernícola en los tubos volcánicos del Valle de Güimar. Publicaciones de la Federación Territorial Canaria de Espeleología, 1, 1-70.

Martínez Méndez, F. 1966. El extinto múrido gigante Canariomys bravoi CRUS. \& PET. Sus características anatómicas y su evolución. Tesina de Lic. Univ. Barcelona, 1-139 (sin publicar).

Mateo, J. A. and López-Jurado, L. F. 1992. Study of dentition in lizards from Gran Canaria Island (Canary Islands) and its ecological and evolutionary significance. Biological Journal of Linnean Society, 46, 39-48.

Meco Cabrera, J. 1992. Los Ovicaprinos de Villaverde. Estudios Prehispánicos. Dirección General de Patrimonio Histórico, 2, $1-167$.

Meco, J. and Petit-Maire, N. 1986. El Cuaternario reciente de Canarias. Le Quaternaire recent des Canaries. Las Palmas, Marsaille, 1-94.

Mertens, R. 1942. Lacerta goliath n. sp., eine ausgestorbene Rieseneidechsen von den Kanaren. Senckenbergiana, 25, 330-339. 
McMinn, M., Jaume, D. and Alcover, J. A. 1990. Puffinus olsoni n. sp.: nova espècie de baldritja recentment extingida provinent de dèposits espeleològics de Fuerteventura i Lanzarote (Illes Canàries, Atlantic Oriental). Endins, 16, 63-71.

Michaux, J., Hutterer, R. and López-Martínez, N. 1991. New fossil faunas from Fuerteventura, Canary Islands: Evidence for a Pleistocene age of endemic rodents and shrews. Compte Rendu de l'Académie des Sciences Paris, 312, série II, 801-806.

Molina, O. M. and Hutterer, R. 1989. A cryptic new species of Crocidura from Gran Canaria and Tenerife, Canary Islands (Mammalia: Soricidae). Bonner Zoologische Beiträge, 40, 85-97.

Montgelard, C. 1991. Systematique moleculaire (Immunologie) de quelques Muridae actuels et fossile. These, Universidad Montpellier II. France, 1-129.

Pérez Torrado, F. J. 1992. Volcanoestratigrafía del Grupo Roque Nublo (Gran Canaria). Tesis Doctoral. Univ. Las Palmas de Gran Canaria, 1-510.

Pieper, H. 1985. The fossil land birds of Madeira and Porto Santo. Bocagiana, 88, 1-6.

Rando, J. C. and Castillo, C. 1993. First data of the diet of the Barn Owl (Tyto cf. alba) in the Quaternary in Fuerteventura (Canary Islands). First Symposium "Fauna and Flora of the Atlantic Islands". Madeira.

Rando, J. C. y Perera, M. A. 1994. Primeros datos de ornitofagía entre los aborigenes de Fuerteventura (Islas Canarias). Archaeofauna, 3, 13-19.
Renz, O., Bernoulli, D. and Hottinger, L. 1992. Cretaceous ammonites from Fuerteventura, Canary Islands. Geological Magazine, 129, 6, 763-769.

Rothe, P. 1964. Fossile Strausseneier auf Lanzarote. Natur und Museum, 94, 175-218.

Rothe, P. 1968. Mesozoische Flysch-Ablagerungen auf der Kanareninsel Fuerteventura. Geologische Rundschau, 58, 314-332.

Rothe, P. 1974. Canary Islands-Origin and Evolution. Naturwissenschaften, 61, 526-533.

Rothe, P. and Schminke, H. U. 1968. Contrasting origins of Eastern and Western Islands of the Canary Archipelago. Nature, 218, 1152-1154.

Rothe, P and Klemmer, K. 1991. Fossil eggs of terrestrial tortoises (Family Testudinidae) from Pliocene calcarenites of Fuerteventura (Canary Islands, Spain). Senckenbergiana lethae, 71, 307-317.

Sauer, E. G. F. 1972. Ratite eggshells and phylogenetic questions. Bonner Zoologische Beiträge, 23, 3-48.

Sauer, E. and Rothe, P. 1972. Ratite eggshells from Lanzarote, Canary Islands. Science, 176, 43-45.

Walker, C. A., Wragg, G. M. and Harrison, C. J. O. 1990. A new shearwater from the Pleistocene of the Canary Islands and its bearing on the evolution of certain Puffinus shearwaters. Historical Biology, 3, 203-224.

Manuscrito recibido: 5 de noviembre, 1994 Manuscrito aceptado: 5 de octubre, 1995 